\title{
Effect of Melilotus suaveolens extract on pulmonary microvascular permeability by downregulating vascular endothelial growth factor expression in rats with sepsis
}

\author{
MING-WEI LIU ${ }^{1}$, MEI-XIAN SU ${ }^{2 *}$, WEI ZHANG ${ }^{1 *}$, YUN HUI WANG $^{1}$, \\ LAN-FANG QIN $^{1}, \mathrm{XU}_{\text {LIU }}^{3}$, MAO-LI TIAN ${ }^{1}$ and CHUAN-YUN QIAN ${ }^{1}$
}

\author{
${ }^{1}$ Department of Emergency, The First Hospital Affiliated To Kunming Medical University, Kunming, Yunnan 650032; \\ ${ }^{2}$ Surgical Intensive Care Unit, The Second Hospital Affiliated To Kunming Medical University, Kunming, Yunnan 650106; \\ ${ }^{3}$ Department of Infectious Diseases, Yan'an Hospital Affiliated To Kunming Medical University, \\ Kunming, Yunnan 650051, P.R. China
}

Received December 7, 2013; Accepted May 23, 2014

DOI: $10.3892 / \mathrm{mmr} .2015 .3146$

\begin{abstract}
A typical indicator of sepsis is the development of progressive subcutaneous and body-cavity edema, which is caused by the breakdown of endothelial barrier function, leading to a marked increase in vascular permeability. Microvascular leakage predisposes to microvascular thrombosis, breakdown of microcirculatory flow and organ failure, which are common events preceding mortality in patients with severe sepsis. Melilotus suaveolens (M. suaveolens) is a Traditional Tibetan Medicine. Previous pharmacological studies have demonstrated that an ethanolic extract of M. suaveolens has powerful anti-inflammatory activity and leads to an improvement in capillary permeability. However, the mechanisms underlying its pharmacological activity remain elusive. The present study aimed to assess the impact of $M$. suaveolens extract tablets on pulmonary vascular permeability, and their effect on regulating lung inflammation and the expression of vascular endothelial growth factor (VEGF) in the lung tissue of rats with sepsis. A cecal ligation and puncture (CLP) sepsis model was established for both the control and treatment groups. $\sim 2 \mathrm{~h}$ prior to surgery, $25 \mathrm{mg} / \mathrm{kg}$ of $M$. suaveolens extract tablet was
\end{abstract}

Correspondence to: Professor Ming-Wei Liu, Department of Emergency, The First Hospital Affiliated To Kunming Medical University, 295 Xichang Road, Kunming, Yunan 650032, P.R. China E-mail: 1mw2004210@163.com

\section{${ }^{*}$ Contributed equally}

Abbreviations: VEGF, vascular endothelial growth factor; NF- $\kappa \mathrm{B}$, nuclear factor kappa B; IL-6, interleukin-6; TNF- $\alpha$, tumor necrosis factor- $\alpha$; LPS, lipopolysaccharide; PCR, polymerase chain reaction; SIRS, systemic inflammatory response syndrome; CLP, cecal ligation and puncture

Key words: sepsis, melilotus extract tablets, vascular endothelial growth factor, microvascular permeability, rats administered to the treatment group. Polymerase chain reaction and western blot analyses were used to assess the expression of nuclear factor (NF)- $\kappa \mathrm{B}$ and VEGF in the lung tissue, and ELISA was applied to detect changes in serum tumor necrosis factor- $\alpha$ as well as interleukins (IL) $-1,-4,-6$, and -10 . The lung permeability, wet/dry weight ratio and lung pathology were determined. The results demonstrated that in the lung tissue of CLP-rats with sepsis, M. suaveolens extract inhibited the expression of $\mathrm{NF}-\kappa \mathrm{B}$, reduced the inflammatory response and blocked the expression of VEGF, and thus significantly decreased lung microvascular permeability. The effects of $M$. Suaveolens extract may be of potential use in the treatment of CLP-mediated lung microvascular permeability.

\section{Introduction}

Sepsis, a systemic inflammatory response syndrome caused by infection, is known to be accompanied by the presence of bacteria, which may arise from a highly virulent focus of infection. Sepsis may often cause acute lung injury (ALI) $(1,2)$. When ALI occurs, cytokines, chemokines, adhesion molecules and other inflammatory mediators are produced in the endothelial cells activated within the pulmonary vasculature, which destroys the integrity of pulmonary vascular endothelial cells. This leads to increased permeability of capillaries in the lung, and consequent pulmonary edema (1), which results in acute respiratory distress syndrome, multiple organ failure, high mortality and other major problems in intensive care. The reduction of pulmonary capillary permeability is therefore of marked clinical importance. Vascular endothelial growth factor (VEGF) is one of the most important regulatory factors during vascular formation. Upon activation of inflammation, alveolar macrophages and neutrophils are able to release a large amount of VEGF $(3,4)$, which most commonly increases the permeability of post-capillary venules. In vitro studies have demonstrated that the effect of VEGF on increasing vascular permeability is 20,000 times more potent than that of histamine $(5,6)$. In the early stage of ALI, neutrophils, monocytes, macrophages and platelets activated by inflammation synthesize 
and release large amounts of VEGF, leading to high vascular permeability and consequently to pulmonary edema (6). Melilotus suaveolens Ledeb (M. suaveolens), a Traditional Tibetan Medicine also known as Melilotus suavcolen or wild alfalfa, is a bitter, 'cold-tasting' herb which has proven to be effective in fever reduction, detoxification, anti-inflammatory and drying limbs ichor (7). It is therefore traditionally applied to treat a range of illnesses, including spleen disease, twisted intestinal fever, diphtheria and tonsillitis (8). Currently, there are few published studies on the effects of $M$. suaveolens. Pharmacological studies have demonstrated that an ethanolic extract from $M$. suaveolens has a powerful anti-inflammatory activity (9), which inhibited formaldehyde and propylene glycol-induced capillary permeability, and was effective in the improvement of blood circulation (10). Therefore, downregulating the expression of VEGF in the lung may be a mechanism by which $M$. suaveolens reduces pulmonary capillary permeability. The aim of the present study was to investigate the mechanism of action by which $M$. suaveolens affects CLP-induced pulmonary capillary permeability in rats, and to establish whether this effect occurs by regulating VEGF expression.

\section{Materials and methods}

Mice. Male Sprague-Dawley mice were purchased from Kunming Medical University Laboratory Animal Center (Kunming, China). All of the mice were housed in the Kunming Medical University Animal Care Facility and were maintained under pathogen-free conditions. The mice were 8-9 weeks of age at the initiation of the experiment and were maintained on a standard laboratory diet and water ad libitum. The experimental procedures were approved by the Committee of Animal Experimentation of the Kunming Medical University (Kunming, China).

Reagents. A reverse transcription reaction kit was purchased from Takara Biotechnology Co. Ltd. (Dalian, China); TRIzol was from Invitrogen Life Technologies (Carlsbad, CA, USA) and electrophoresis reagents were from Promag Co. (Ningbo, China); an RT Reaction kit was obtained from Takara Biotechnology Co. Ltd (Dailan, China); a PCR Amplification Reagent kit and the 100 bp DNA ladder marker were obtained from Sangon Biological Engineering Co. Ltd. (Shanghai, China); GAPDH was obtained from Santa Cruz Biotechnology, Inc. (Santa Cruz Biotechnology, Inc., Santa Cruz, CA, USA); rabbit anti-mouse NF- $\kappa$ B and VEGF polyclonal antibodies were purchased from Wuhan Boster Biological Technology, Ltd. (Wuhan, China); M. suaveolens Extract Tablets were from Seiko Eiyo Yakuhin Co. Ltd. (Osaka, Japan); fluorescein isothiocyanate (FITC)-albumin and hexadecyl-trimethyl-ammonium bromide were purchased from Sigma-Aldrich (St. Louis, MO, USA) and SYBR green I was obtained from Biotium (Hayward, CA, USA). The Oligo (dT18) and primers were synthesized by Shanghai Invitrogen (Shanghai, China). The dNTP was obtained from Promega Corp. (Madison, WI, USA).

Animal model of sepsis. All of the studies were performed on rats with an average weight of $40.4 \mathrm{~g}$. To induce sepsis, the rats were anesthetized with isoflurane (4\% induction, $2 \%$ maintenance) and placed on a warming pad. Following laparotomy, the cecum was exteriorized, and the membrane between the cecum and the mesentery was carefully cut to release the cecum. The cecum was ligated $1.5 \mathrm{~cm}$ from the tip or just below the ileocecal valve with 4-0 silk. Two punctures were made with an 18-gauge needle and $1 \mathrm{~mm}$ of fecal material was expressed from the punctures. The incision was sutured in two layers with 4-0 silk. In the sham animals, the cecum was located but neither ligated nor punctured. The animals were resuscitated with $3 \mathrm{ml} / 100 \mathrm{~g}$ body weight normal saline subcutaneously immediately following surgery.

Grouping and treatment. According to a random number table, 88 rats were randomly divided into four groups: Normal control group, sham operation group (sham group), sepsis model group [(untreated) sepsis group] and M. suaveolens treatment group (treatment group), with 22 rats in each group. The model group and treatment group were induced by cecal ligation and puncture (CLP) and, $2 \mathrm{~h}$ prior to surgery, were administered the $M$. suaveolens extract via tube, at a dose of $25 \mathrm{mg} / \mathrm{kg}$ every $8 \mathrm{~h}$. The normal control group, sham group and (untreated) sepsis group were subject to treatment with the same volume of normal saline. A total of 22 rats in each group were anesthetized using ether at each of the following time-points $24 \mathrm{~h}$ post-surgery. Subsequently, the right internal carotid artery was isolated. Blood was collected via the orbital sinus. EDTA was used as an anti-coagulant, and the plasma was isolated by centrifugation at $10,000 \mathrm{x} \mathrm{g}$ for $5 \mathrm{~min}$. All the animals were sacrificed $24 \mathrm{~h}$ following surgery via anesthesia with ether and lung tissues were collected, washed with saline solution, dried with filter paper and weighed. The plasma and tissues were stored at $-20^{\circ} \mathrm{C}$ for subsequent experiments.

$R N A$ isolation and quantitative polymerase chain reaction ( $q P C R)$. The left lung tissues were homogenized in TRIzol reagent using a Mixer Mill301 (Tianjin Tian Chang Technology Co., Ltd., Tianjin, China). The total RNA was extracted using TRIzol reagent according to the manufacturer's instructions and quantified spectrophotometrically. A total of $2 \mu \mathrm{g}$ RNA from each sample was added to a total volume of $25 \mu \mathrm{l}$ reaction mixture containing $2.5 \mu \mathrm{M}$ oligo (dT) primer (Promega Corp.; cat. no. C110A), and 200 U Molony murine leukemia virus reverse transcriptase (M-MLV; Promega Corporation; cat. no. M5314). The reaction was initiated by incubating the reaction mixture for $1 \mathrm{~h}$ at $42^{\circ} \mathrm{C}$ for reverse transcription and stopped by heating for $10 \mathrm{~min}$ at $70^{\circ} \mathrm{C}$. An aliquot $(0.5 \mu \mathrm{l})$ of each reverse transcription product was added to $20 \mu \mathrm{l}$ reaction mixture containing LightCycler-FastStart DNA Master SYBR Green I, $0.5 \mu \mathrm{M}$ of each primer corresponding to mouse NF- $\kappa \mathrm{B}$ and VEGF or GAPDH, and $4 \mathrm{mM} \mathrm{MgCl}_{2}$ to amplify the genes in a LightCycler (Roche, Mannheim, Germany). For reverse transcription PCR, $1 \mu \mathrm{g}$ of total RNA from each sample was resuspended in $25 \mu$ l final volume of reaction buffer. GAPDH was used as an internal control. The following primers were used for PCR: VEGF forward primer, 5'-GCTCTCTTGGGTGCACTGGA-3' and reverse primer, 5'-CACGCCTTGGCTTGTCACCA-3'; NF- $\kappa$ B forward primer, 5'-GCACGGATGACAGAGGCGTGTATAAGG-3' and reverse 
primer, 5'-GGCGGATGATCTCCTTCTCTCTGTCTG-3'; and GAPDH forward primer, 5'-AAT GCA TCC TGC ACC ACC AA-3' and reverse primer, 5'-GTA GCC ATA TTC ATT GTC ATA-3'. Following pre-incubation at $95^{\circ} \mathrm{C}$ for $10 \mathrm{~min}$, the PCR was performed as follows: 35 cycles of denaturation at $95^{\circ} \mathrm{C}$ for $15 \mathrm{sec}$, annealing at $60^{\circ} \mathrm{C}$ for $5 \mathrm{sec}$ and elongation at $72^{\circ} \mathrm{C}$ for $12 \mathrm{sec}$. The $2^{-[\Delta \Delta \mathrm{Ct}]}$ method was used to compare the mRNA expression levels of genes in the experimental groups with the control groups.

Western blot analysis. The lung tissues were snap-frozen in liquid nitrogen, pulverized and resuspended in ice-cold lysis buffer (Solarbio Science \& Technology, Beijing, China). Protein concentrations were determined with the Bradford method. Lysates were allowed to solubilize on ice for $30 \mathrm{~min}$ and particulate mass was removed by centrifugation $(15,000 \mathrm{x} \mathrm{g})$ for $15 \mathrm{~min}$ at $4^{\circ} \mathrm{C}$. The supernatants were analyzed by SDS-PAGE. Primary antibodies used included rabbit anti-VEGF monoclonal antibody (1:400), rabbit anti-NF-кB65 monoclonal antibody (1:400) (Boster Biological Technology, Ltd) and mouse anti- GAPDH monoclonal antibody (1:400) (Santa Cruz Biotechnology, Inc). The secondary antibodies were horseradish peroxidase-labeled antibodies (Pierce; Thermo Scientific, Rockford, IL, USA). The blots were processed for enhanced chemifluorescence using a Pierce ECL Western blotting substrate (Pierce; Thermo Scientific).

Immunohistochemistry. Immunostaining was performed on the lung sections following antigen retrieval using Retrievagen A (Zymed Laboratories, Inc., San Francisco, CA, USA) at $100^{\circ} \mathrm{C}$ for $20 \mathrm{~min}$, and quenching endogenous peroxidases with $3 \% \mathrm{H}_{2} \mathrm{O}_{2}$. The sections were blocked with $2 \%$ bovine serum albumin (BSA) in phosphate-buffered saline (PBS) followed by staining with primary anti-VEGF- $\alpha$ and anti-NF-kBp65 (BD Pharmingen, San Jose, CA, USA) at room temperature for $1 \mathrm{~h}$. The sections were washed and following application of secondary antibody (R\&D Systems, Minneapolis, MN, USA), tissues were developed using Vectastain ABC (Vector Laboratories, Inc., Burlingame, CA, USA) and 3,3'-diaminobenzidine (Vector Laboratories, Inc.). Following staining, five high-power fields (x200) were randomly selected in each slide, and the average proportion of positive expression in each field was counted using the true color multi-functional cell image analysis management system (Image-Pro Plus; Media Cybernetics, Rockville, MD, USA), and expressed as positive unit (PU).

Cytokine and VEGF measurements in bronchoalveolar lavage (BAL) and plasma. Mice were sacrificed after $24 \mathrm{~h}$ of treatment, and BAL was performed via the tracheal catheter in the right lung lobes using $0.8 \mathrm{ml}$ PBS; the withdrawn fluid was centrifuged, and the supernatant was snap frozen and stored at $-80^{\circ} \mathrm{C}$ for further use. Aliquots of BAL fluid and plasma were detected in duplicate by ELISA (ELISA kit offered by Glory Science Co., Ltd., Del Rio, TX, USA) kits for tumor necrosis factor- $\alpha$ (TNF- $\alpha$ ), interleukin (IL)-1 $\beta$, IL- 6 and IL-10 according to the manufacturer's instructions.

Pulmonary vascular permeability assays. Two hours prior to sacrification, FITC-labeled albumin ( $5 \mathrm{mg} / \mathrm{kg}$ body weight) was administered via tail-vein injection at 6 and $24 \mathrm{~h}$. Immediately following sacrification, the lungs were lavaged three times with PBS ( $0.5 \mathrm{ml}$ per lavage) and the samples combined. Fluid recovery was $\sim 95 \%$. The BAL samples were centrifuged at $3,000 \mathrm{x}$ g for $10 \mathrm{~min}$. FITC fluorescence in the BAL fluid was measured using a 960CRT computer controlled fluorescence spectrophotometer (Shanghai Tiancheng Technology Co., Ltd., Shanghai, China) with excitation at $484 \mathrm{~nm}$ and emission at $510 \mathrm{~nm}$.

Wet/dry (W/D) lung weight ratio and water content. W/D weight ratio was used as an index of tissue water content. $24 \mathrm{~h}$ following administration of $M$. suaveolens extract, the animals were anesthetized using ketamine $(80 \mathrm{mg} / \mathrm{Kg}$ i.p.) and xylazine $(20 \mathrm{mg} / \mathrm{Kg}$ i.p.), sacrificed and lungs were excised en bloc. The lung lobes were cut, blot dried and placed on pre-weighed glass plates. The wet weight of the tissue was determined immediately. The tray with the tissue was then baked in an oven at $55^{\circ} \mathrm{C}$ for $72 \mathrm{~h}$ to obtain a constant weight. After the dry weight of the tissue was determined, the wet/dry (W/D) lung weight ratio was calculated. The lung water content was calculated as the wet weight minus dry weight and wet weight ratio of lung tissue multiplied by $100 \%$.

Pathological observation of lung tissues. The middle lobe of the right lung was fixed by infusing $10 \%$ formaldehyde solution in the same pressure, and the inflation of the lung was kept uniform. The tissue was then embedded in paraffin wax, cut into sections and stained with hematoxylin-eosin (H\&E). Pathological changes in the tissues were observed using optical microscopy. Lung injury, based on infiltration of inflammatory cells, pulmonary interstitial and alveolar edema, damage to alveolar structure and degree of fibrosis were assessed using the grading system reported by Szapiel et al (11). ALI was scored as follows (12): i) Alveolar congestion; ii) hemorrhage; iii) infiltration or aggregation of neutrophils in airspace or vessel wall, and iv) thickness of alveolar wall/hyaline membrane formation. Each item was scored on a five-point scale as follows: 0 , minimal damage; 1 , mild damage; 2 , moderate damage; 3 , severe damage; and 4 , maximal damage. Repeatedly measured data were statistically analyzed using analysis of variance (ANOVA).

Statistical analysis. Statistical analysis was performed with the SPSS version 15.0 (SPSS, Inc., Chicago, IL, USA). Data were analyzed for normality using the Kolmogorov-Smirnov method, and the normally distributed data were expressed as the mean \pm standard deviation. To compare the normally distributed data between each group, one-way ANOVA followed by the Student-Newman-Keul's post-hoc test was employed. $\mathrm{P}<0.05$ was considered to indicate a statistically significant difference.

\section{Results}

M. suaveolens extract downregulates the expression of VEGF and $N F-\kappa B$ in lung tissue. To investigate the underlying mechanism of the effect of M. suaveolens extract on CLP, the mRNA and protein expression of VEGF and NF- $\mathrm{kB}$ were measured by qPCR and western blot analysis, respectively. In vivo, the mRNA and protein expression levels of VEGF 
A a

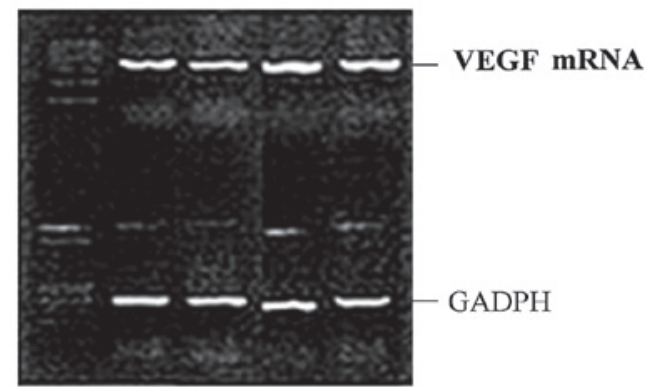

B

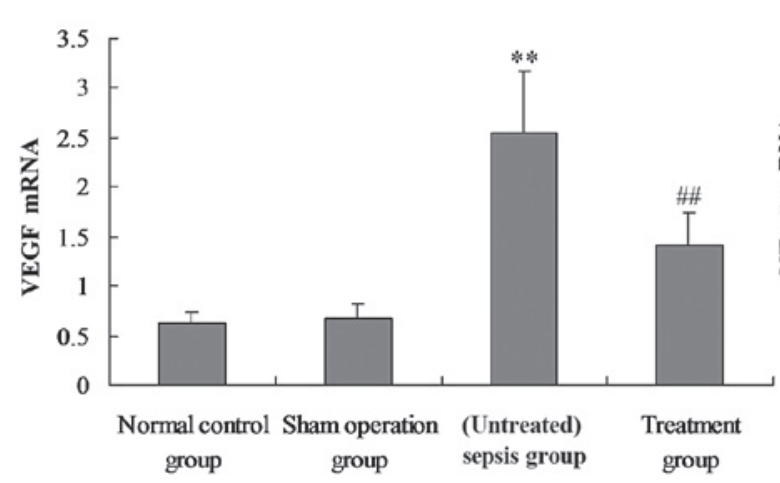

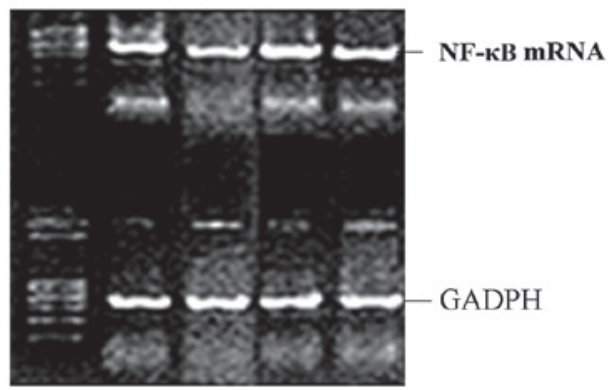

b

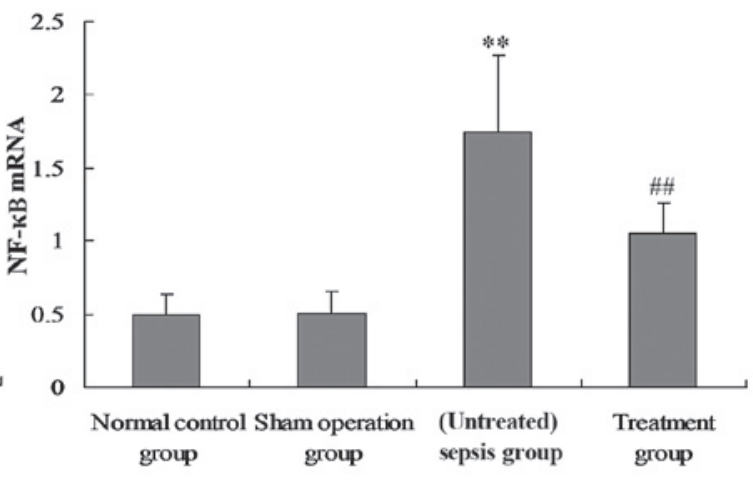

Figure 1. M. suaveolens extract blocked the expression of VEGF and NF- $\mathrm{kB}$ mRNA in lung tissue. Groups of mice were challenged with cecal ligation and puncture and treated with M. suaveolens extract $24 \mathrm{~h}$ later. The left lung tissues were homogenized and total RNA was extracted using TRIzol reagent and assayed by quantitative polymerase chain reaction. (A) Representative gels assessing (a) VEGF and (b) NF-кB levels are demonstrated. Lane 1, marker; lane 2, normal control group; lane 3, sham operation group; lane 4, (untreated) sepsis group and lane 5, treatment group. (B) Statistical summary of the densitometric analysis of VEGF and NF- $\kappa$ B mRNA expression in rats from the four groups; (a) VEGF mRNA and (b) NF- $\kappa B$ mRNA. Data are represented as the mean \pm standard deviation of one experiment consisting of three replicates. ${ }^{* *} \mathrm{P}<0.01$ vs. sham operation and normal control groups; ${ }^{\# \#} \mathrm{P}<0.01 \mathrm{vs}$. (untreated) sepsis group. VEGF, vascular endothelial growth factor; NF-kB, nuclear factor kappa B.

A

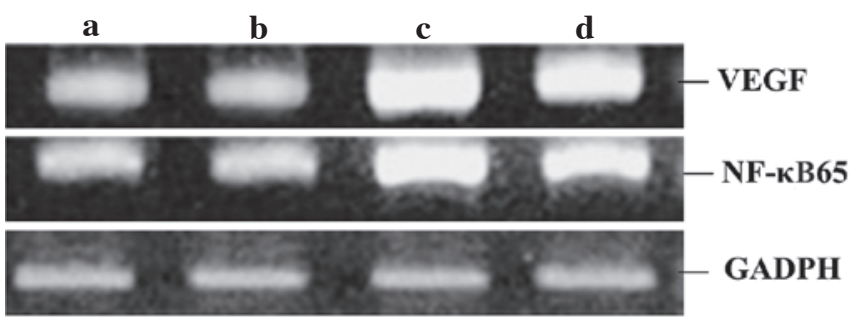

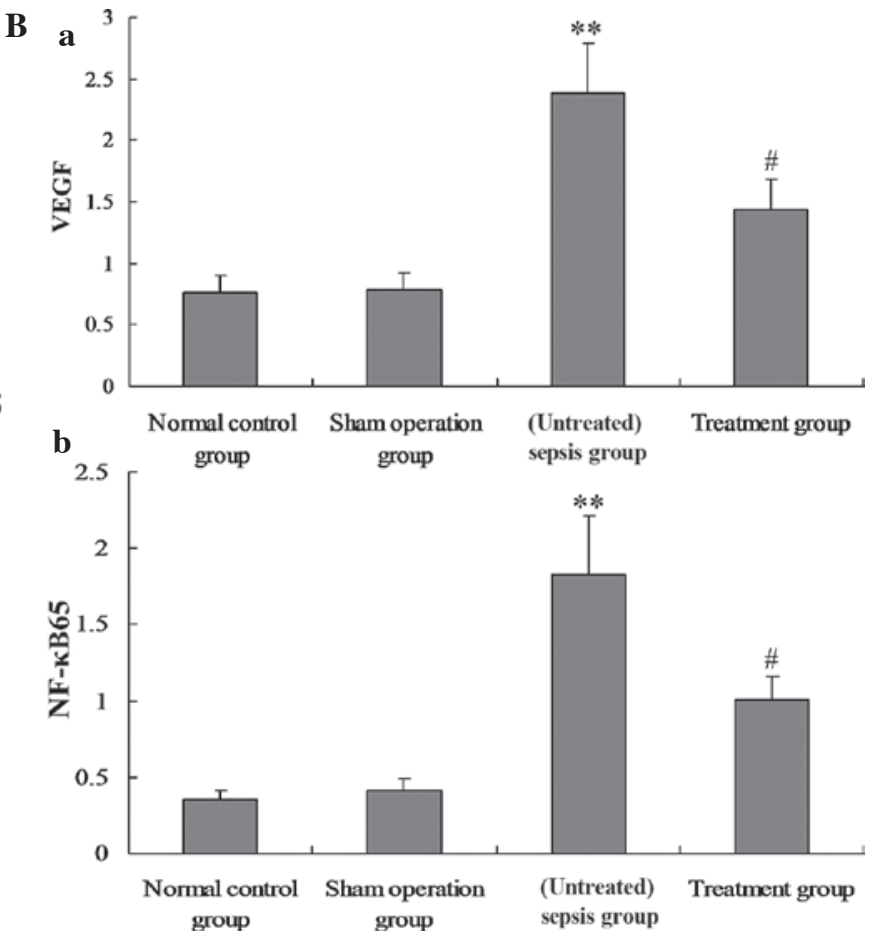

Figure 2. Effect of M. suaveolens extract on the expression of VEGF and NF- $\mathrm{kB} 65$ protein in lung tissue. Groups of mice were challenged with cecal ligation and puncture and treated with $M$. suaveolens extract $24 \mathrm{~h}$ later. The expression of VEGF, NF- $\kappa \beta 65$ and GAPDH was detected by western blotting using specific antibodies. GAPDH protein was used an internal control. (A) Representative western blot analysis demonstrated the levels of VEGF and NF- $\mathrm{kB} 65$ protein expression in rats from the four groups; (a) normal control group; (b) sham operation group; (c) (untreated) sepsis group and (d) treatment group. (B) Quantification of the blots by densitometric analysis of (a) VEGF and (b) NF-kB65 protein expression in rats from the four groups. Data are presented as the mean \pm standard deviation of one experiment consisting of three replicates. ${ }^{* *} \mathrm{P}<0.01$ vs. the sham operation group and normal control group; ${ }^{*} \mathrm{P}<0.05$ vs. (untreated) sepsis group. VEGF, vascular endothelial growth factor; NF-кB, nuclear factor kappa B. 
A

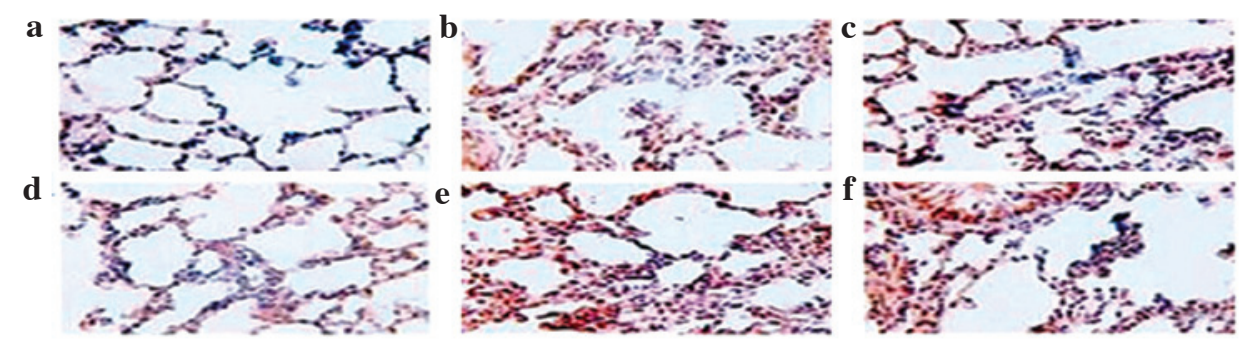

B
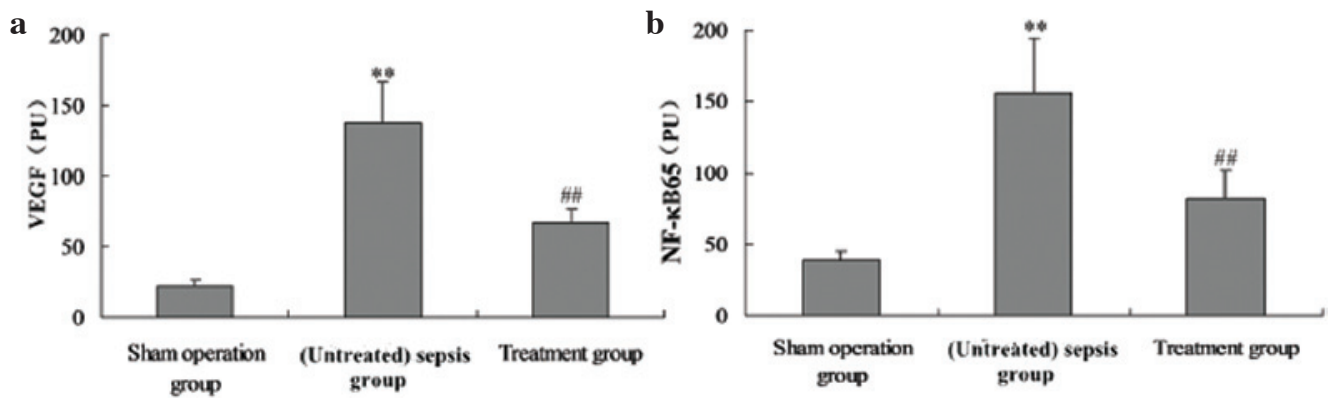

Figure 3. Effect of M. suaveolens extract on the protein expression of VEGF and NF- $\mathrm{kB} 65$ in rat lungs $24 \mathrm{~h}$ following cecal ligation and puncture-induced acute lung injury. Immunostaining was performed on lung sections following antigen retrieval using Retrievagen. (A) Representative immunostaining revealed VEGF and NF-K $\beta 65$-positive expression in rats from the four groups: (a-c) Expression of positive VEGF in the (a) sham operation group; (b) control group; (c) treatment group); (d-f) Expression of positive NF-kB65 in the (d) sham operation group; (e) (untreated) sepsis group; (f) treatment group (magnification, x200). (B) Quantification of the images by densitometric analysis of (a) VEGF and (b) NF-kB-positive protein expression in rats from four groups. All values are expressed as the mean \pm standard deviation. ${ }^{* *} \mathrm{P}<0.01$ vs. the sham operation group; ${ }^{\# \#} \mathrm{P}<0.01$ vs. (untreated) sepsis group. VEGF, vascular endothelial growth factor; NF- $\mathrm{kB}$, nuclear factor kappa B.
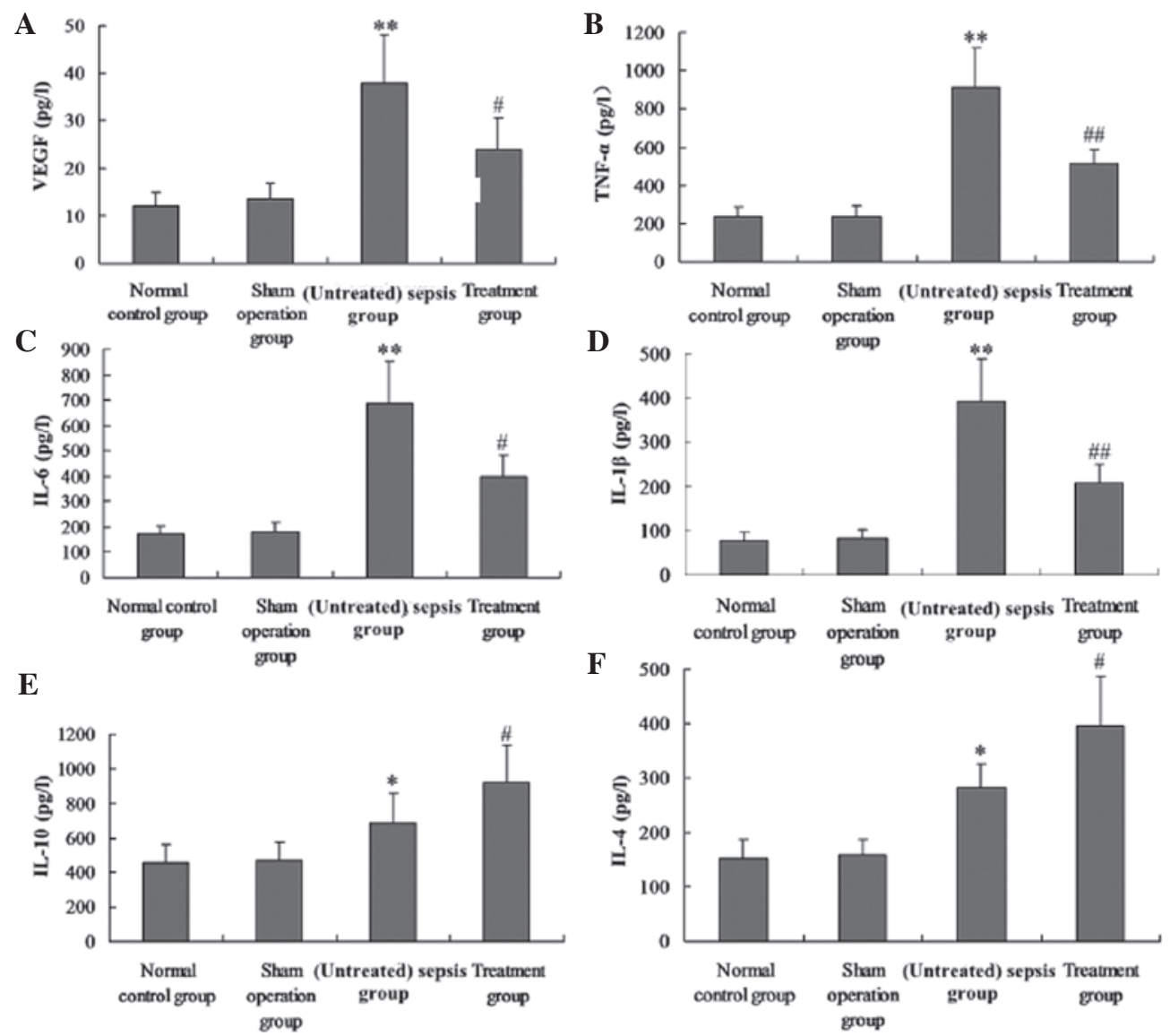

Figure 4. Effect of $M$. suaveolens extract on plasma levels of VEGF, TNF- $\alpha$, IL-6, IL-1 $\beta$, IL-4 and IL-10 levels in plasma. Groups of mice were challenged with cecal ligation and puncture, and treated with M. suaveolens extract $24 \mathrm{~h}$ later. (A) VEGF, (B) TNF- $\alpha$, (C) IL-6, (D) IL-1 $\beta$, (E) IL-10 and (F) IL-4 levels in plasma were determined by ELISA. Data are presented as the mean \pm standard deviation of one experiment consisting of three replicates. ${ }^{*} \mathrm{P}<0.05,{ }^{* *} \mathrm{P}<0.01 \mathrm{vs}$. the sham operation group and normal control group; ${ }^{\sharp} \mathrm{P}<0.05,{ }^{\# \#} \mathrm{P}<0.01$ vs. (untreated) sepsis group. VEGF, vascular endothelial growth factor; $\mathrm{NF}-\mathrm{\kappa} B$, nuclear factor kappa B; TNF- $\alpha$, tumor necrosis factor- $\alpha$; IL, interleukin. 

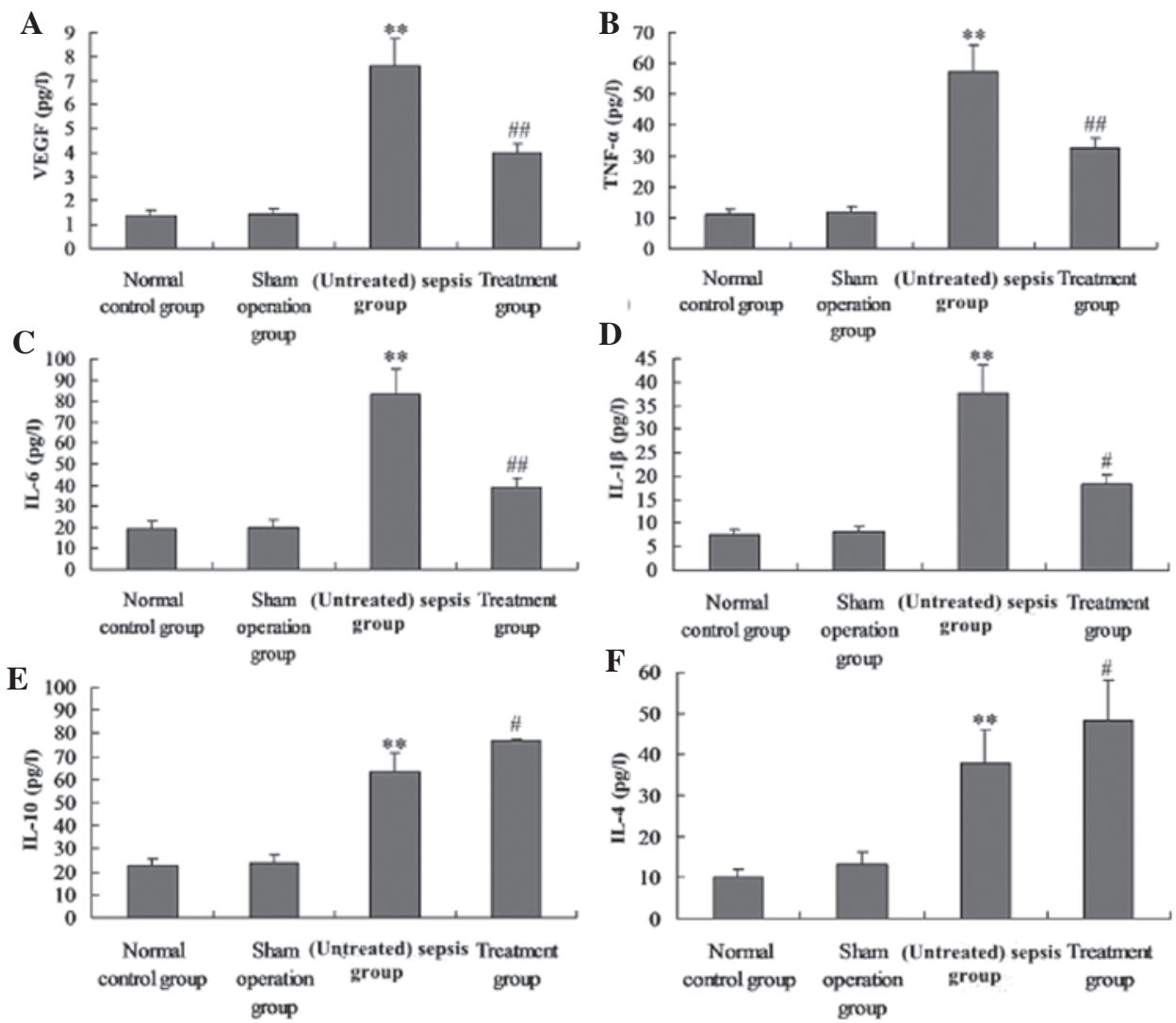

Figure 5. Administration of M. suaveolens extract attenuated lipopolysaccharide-induced pulmonary inflammation. Groups of mice were challenged with cecal ligation and puncture, and treated with M. suaveolens extract 24 h later. (A) VEGF, (B) TNF- $\alpha$, (C) IL-6, (D) IL-1 $\beta$, (E) IL-10 and (F) IL-4 levels in bronchoalveolar lavage were determined by ELISA. Data are presented as the mean \pm standard deviation of one experiment consisting of three replicates. ${ }^{* *} \mathrm{P}<0.01 \mathrm{vs}$. the sham operation group and normal control group; ${ }^{\# \mathrm{P}}<0.05,{ }^{\# \#} \mathrm{P}<0.01$ vs. (untreated) sepsis group. VEGF, vascular endothelial growth factor; $\mathrm{NF}-\mathrm{kB}$, nuclear factor kappa B; TNF- $\alpha$, tumor necrosis factor- $\alpha$; IL, interleukin.

and NF- $\mathrm{\kappa B}$ in the rat lung demonstrated significant increases during CLP-induced ALI (P<0.05; Figs. 1 and 2); however, these were significantly decreased in response to the administration of M. suaveolens extract $(\mathrm{P}<0.05$; Figs. 1 and 2). A positive correlation was evident between the levels of VEGF and NF- $\kappa B$ mRNA $(r=0.852, \mathrm{P}<0.05)$ and between VEGF and NF- $\kappa B$ protein expression $(r=0.794, \mathrm{P}<0.01)$.

Effect of M. suaveolens extract on lung localization of VEGF and $N F-\kappa B 65$ in CLP-induced ALI. Immunohistochemical analysis was used to determine the distribution of VEGF and NF-kB65 in rat lung $24 \mathrm{~h}$ following CLP or saline treatment. Positively immunostained cells appeared brown (Fig. 3A). VEGF and NF- $\mathrm{BB} 65$ were localized to the alveolar epithelium. The number of cells expressing VEGF and NF- $\kappa$ B65 was significantly increased in CLP-induced ACI, and this was significantly reduced by treatment with $M$. suaveolens extract (Fig. 3A and B).

M. suaveolens extract decreases pro-inflammatory cytokines and VEGF production in CLP-induced rats. Serum and BAL were collected $24 \mathrm{~h}$ after the animals received CLP to evaluate the levels of VEGF, TNF- $\alpha$, IL-1 $\beta$, IL-6, IL-4 and IL-10. CLP caused a significant acute systemic inflammatory response and pulmonary capillary permeability, as demonstrated by the increased serum and BAL concentrations of the pro-inflammatory mediators TNF- $\alpha$, IL-1 $\beta$, IL- 6 and VEGF. The presence of M. suaveolens extract reduced the increase of all of these pro-inflammatory mediators. CLP also caused an increase in the serum and BAL concentration of the anti-inflammatory cytokines IL-10 and IL-4. This change in IL-10 and IL-4 concentration was enhanced by the administration of $M$. suaveolens extract (Fig. 4E and F; Fig. 5E and F).

Effect of M. suaveolens extract on pulmonary vascular permeability. M. suaveolens extract significantly reduced the CLP-induced increases in i.v. administered FITC-labeled albumin in BAL, as well as the W/D lung weight ratio and the water content of the lung tissue $(\mathrm{P}<0.05$; Fig. 6A-C). The effects of CLP on pulmonary vascular permeability were also significantly suppressed by M. suaveolens extract ( $\mathrm{P}<0.05$; Fig. 6A-C).

Effect of M. suaveolens extract on histological parameters of $A C I$. The lung tissue was significantly injured as indicated by the presence of intra-alveolar exudate, edema and inflammatory cell infiltration in the control group (Fig. 7A), and an increase in lung injury score $(\mathrm{P}<0.05$, Fig. $7 \mathrm{~B})$. M. suaveolens extract significantly attenuated CLP-induced pathologic changes, demonstrating a significant decrease in lung injury score.

\section{Discussion}

Numerous studies have demonstrated that the animal model of sepsis induced by the CLP method is highly stable 


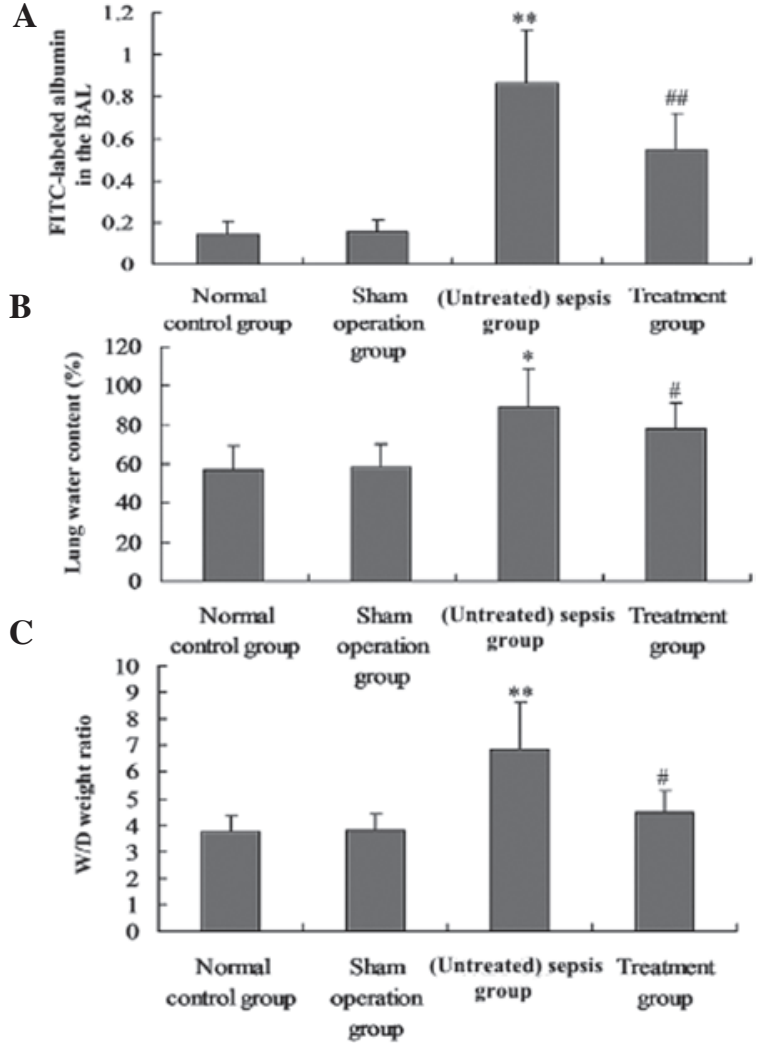

Figure 6. Administration of M. suaveolens extract reduces CLP-induced lung permeability. Rats were treated as indicated and (A) FITC-labeled albumin in the bronchoalveolar lavage fluid, (B) water content of lung tissue and (C) W/D lung weight ratio were determined $24 \mathrm{~h}$ following CLP challenge. Data are presented as the mean \pm standard deviation of one experiment consisting of three replicates. ${ }^{*} \mathrm{P}<0.05,{ }^{* *} \mathrm{P}<0.01$ vs. the sham operation and normal control groups ${ }^{\#} \mathrm{P}<0.01$ vs. (untreated) sepsis group. VEGF, vascular endothelial growth factor; NF- $\kappa \mathrm{B}$, nuclear factor kappa B; TNF- $\alpha$, tumor necrosis factor- $\alpha$; IL, interleukin; CLP, cecal ligation and puncture; W/D, wet/dry; FITC, fluorescein isothiocyanate.

and reproducible, and is applicable as a model of human sepsis (13). It is therefore currently regarded as the 'gold standard' for the study of sepsis. These animals demonstrate a hyperdynamic circulation and high metabolism in the early stage and a low dynamic circulation state at the later stage resembling the human condition; therefore, this is regarded as the sepsis model with the strongest clinical relevance (13). As a result, this CLP animal model of sepsis was adopted for the present study.

When sepsis occurs, a large number of inflammatory mediators are released(1,14-17); endothelial cells are impaired and contract, and the distance between endothelial cells is enlarged $(14,15)$, all of which result in protein-rich fluid entering the mesenchymal cells from the blood vessels. This leads to a drop in the vascular endothelial colloidal osmotic pressure and an increase of colloidal osmotic pressure for tissue clearance, resulting in the presence of more water molecules among the organelles $(16,17)$, and thus the development of interstitial edema. The diffusion distance for oxygen molecules from the blood capillary to tissue organs is increased, further aggregating hypoxia and leading to organ dysfunction $(15,16)$. Furthermore, hypoxemia and hypoxia form a vicious cycle, resulting in further hypoxia-induced injury within the endothelial cells, leading to further capillary leakage (14). In the present study, CLP markedly enhanced the expression of pro-inflammatory mediators, including TNF- $\alpha$ and IL- 6 , and anti-inflammatory mediators, including IL-10, and increased lung capillary leakage. However, the administration of $M$. suaveolens extract significantly inhibited the expression of pro-inflammatory mediators, elevated the levels of anti-inflammatory mediators and reduced lung capillary leakage. Inhibition of inflammatory mediators by M. suaveolens therefore reduced the permeability of the lung capillary wall and reduced CLP-induced lung injury.

VEGF, a protein first obtained by isolation from in vitro cultures of bovine pituitary follicles in 1989, has specific mitogenic effects on vascular endothelial cells which promote endothelial cell proliferation, increase microvascular permeability and promote the growth of endothelial cells within the blood and lymphatic vessels (18). VEGF is therefore a survival factor existing in endothelial cells. VEGF has been used in numerous fields since its discovery, including as a marker for clinical tumor prognosis, early diagnosis of acute myocardial ischemia and evaluation of bronchial asthma (19). In recent years, its role in sepsis blood capillary leakage has drawn increasing attention $(5,19,20)$. VEGF has a significant role among the known microvascular permeability inducers, and effects $\sim 50,000$-fold of those of histamine have been observed, with activities at concentrations $<1 \mathrm{nmol} / \mathrm{l}$. Such effects are not inhibited by antihistamines, platelet-activating factor inhibitors or other inhibitors of inflammation (5). An excessive increase of vascular permeability may cause blood flow into the tissue, leading to poor blood supply for visceral function, eventually resulting in organ dysfunction. van der Flier et al (19) suggested that an increase in capillary permeability is a key factor in the occurrence and development of sepsis, while VEGF is the key molecule for controlling vascular permeability, and is therefore a potential factor that leads to inflammation-associated capillary permeability. For this reason, VEGF was used as a measurement index in the present study. The results indicated that increased expression of NF- $\kappa \mathrm{B}$ promotes the expression and production of VEGF, and significantly aggravates pathological lung damage in rats with sepsis.

It has been demonstrated that through its involvement in the transcription of a variety of cytokine genes, NF- $\mathrm{KB}$ has a complex and important role in regulating the inflammatory network (21). Activated NF- $\kappa \mathrm{B}$ may increase the transcription of numerous cytokines, including TNF- $\alpha$ and IL-1, thus rapidly increasing the quantity of inflammatory factors synthetized by inflammatory cells $(22,23)$. Therefore, blocking the activation of NF- $\mathrm{KB}$ may facilitate controlling the blood capillary permeability of lung tissue, and thus attenuate lung microvascular leakage and ALI in rats with sepsis. It is observed from this study that inhibition of NF- $\mathrm{kB}$ activity by $M$. suaveolens significantly reduced lung inflammatory responses, decreased the expression of VEGF and alleviated capillary permeability in rats with sepsis.

Vascular leakage in multiple organs is a characteristic pathological change in sepsis (24). In the present study, the W/D ratio of the lung was firstly examined. It was identified that salidroside treatment attenuates the development of pulmonary edema, as demonstrated by a significant decrease in lung W/D ratio. Another index of ALI by CLP is the total 

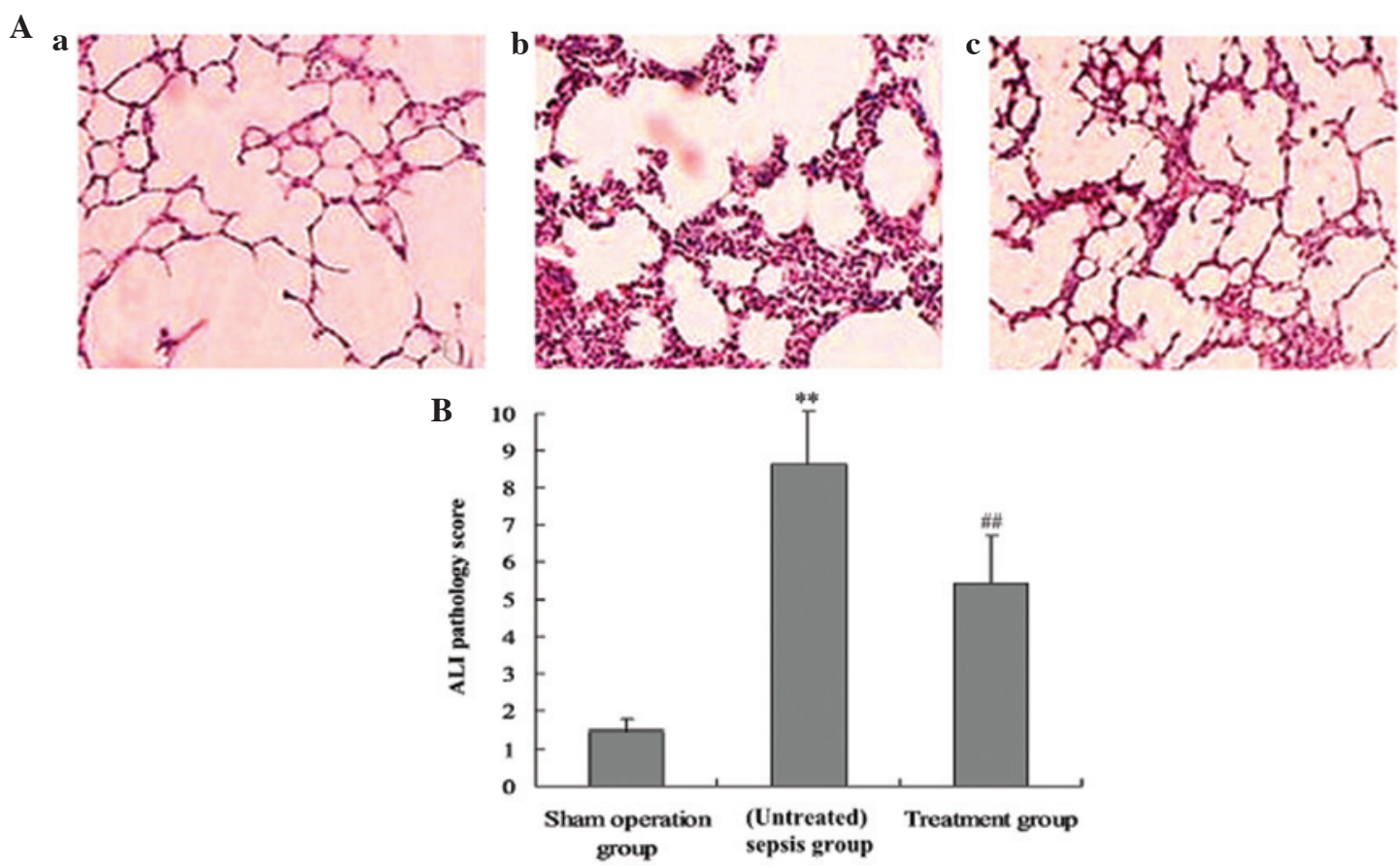

Figure 7. Administration of M. suaveolens extract ameliorated histopathologal changes in the lung tissue of CLP-ALI rats. The groups of rats were treated as described above and histological evaluation of the therapeutic potential of M. suaveolens extract on CLP-induced lung injury was performed $24 \mathrm{~h}$ following CLP challenge. (A) Representative images of hematoxylin and eosin-stained lung sections from three experimental groups (magnification, $\mathrm{x} 400$ ): (a) Sham operation group; (b) (untreated) sepsis group; and (c) treatment group. (B) Lung injury score. ALI pathology score are expressed as the mean \pm standard deviation of one experiment consisting of three replicates. ${ }^{* *} \mathrm{P}<0.01$ vs. the sham operation group; ${ }^{\# \#} \mathrm{P}<0.01$ vs. (untreated) sepsis group. CLP, cecal ligation and puncture; ALI, acute lung injury.

protein concentration in the BAL fluid which indicated epithelial permeability and pulmonary edema. FITC-labeled albumin, a macromolecular marker, is widely used to evaluate pulmonary microvascular permeability (25), and FITC-labeled albumin was therefore injected and assessed in the BAL in the present study. As expected, CLP was identified to cause a significant increase in BAL fluid protein concentration, lung W/D ratio and FITC-labeled albumin. CLP-induced increases in total protein in the BAL fluid, lung W/D ratio and FITC-labeled albumin were inhibited by $M$. suaveolens. In the present study, it was also identified that $M$. suaveolens significantly reduced the accumulation and sequestration of activated macrophages, suppressed formation of pulmonary edema, prevented an increase in septa thickness and ameliorated the lung capillary leakage in rats with sepsis.

Capillary leakage and endothelial dysfunction are increasingly recognized to significantly contribute to organ failure and death in sepsis and systemic inflammation (26-28). Therapeutic targeting of capillary leakage in sepsis and systemic inflammation is therefore considered a highly relevant clinical approach. The pharmacological effects of $M$. suaveolens $(8,29-34)$ indicate that one of the active components, coumarin, is able to reduce capillary permeability and vascular resistance $(8,29)$, increase vein tension and improve circulation $(30,31)$, so that the open arteriovenous anastomosis tubes are closed to reduce local congestion, therefore reducing soft tissue bleeding. Coumarin may also inhibit the release of a variety of vascular active substances (32), suppressing adenosine diphosphate and collagen-induced platelet aggregation as well as the release of 5-hydroxytryptamine, platelet factor 4, thromboxane A2 and platelet-derived growth factor from the platelet $(33,34)$. These effects prevent the loss of serum, maintain normal colloid osmotic pressure and have anti-edema effects, therefore reducing post-traumatic swelling. The tannic acid component of $M$. suaveolens inhibits the synthesis of prostaglandins and other inflammatory mediators, as well as reducing pain and the inflammatory reaction, reducing vascular permeability and leakage from tissue clearance. Other effects include increasing the formation of newborn granulation tissue, promoting wound healing (closing the wound and preventing secondary wound exudate), expanding lymphatic vessels, increasing lymph flow, accelerating lymph circulation and reducing soft tissue swelling. Medicinal chemistry study results have identified that $M$. suaveolens contains coumarin, flavonoids, phenolic acids, saponins and other substances that have effective anti-inflammatory and antibacterial activities (31). Although its effect of reducing the blood capillary leakage has been reported, studies of how this occurs at the molecular level remain limited. The present study revealed that M. suaveolens is able to inhibit the activation and expression of NF- $\mathrm{KB}$ in rats with sepsis, reduce the generation of inflammatory mediators, including TNF- $\alpha$ and IL-6 (with consequent reduction of VEGF expression), therefore decreasing lung capillary permeability and having a protective role in ALI.

Although an ability of $M$. suaveolens to reduce blood capillary leakage has been reported in clinical practice, the molecular biological mechanisms mediating this effect have not been well investigated. The results of the present study suggest a novel compensatory mechanism for the action of $M$. suaveolens in the maintenance of lung vascular permeability under pathological inflammatory conditions, through 
downregulating VEGF expression. To the best of our knowledge, the present study demonstrated for the first time the role of M. suaveolens in mediating anti-inflammatory and barrier protective effects of lung capillary permeability in a model of ALI. Therefore, the beneficial effects of in vivo administration of $M$. suaveolens on the parameters of capillary permeability in lung injury described in this study may represent a novel therapeutic modality for the treatment of CLP-induced lung capillary permeability and ALI.

\section{Acknowledgements}

The authors are grateful to Professor Mei-xian Sun and Professor Lan-fang Qin for their technical assistance.

\section{References}

1. Park SJ, Pai KS, Kim JH and Shin JI: What dose of intravenous immunoglobulin should be administered in Kawasaki disease with suspected systemic capillary leak syndrome? Comment on: shock: an unusual presentation of Kawasaki disease (Eur J Pediatr 2011 Jul; 170(7):941-3). Eur J Pediatr 17:203-204, 2012.

2. Zhao L, Tao JY, Zhang SL, et al: N-butanol extract from Melilotus Suaveolens Ledeb affects pro- and anti-inflammatory cytokines and mediators. Evid Based Complement Alternat Med 7: 97-106, 2010.

3. van Meurs M, Castro P, Shapiro NI, et al: Adiponectin diminishes organ-specific microvascular endothelial cell activation associated with sepsis. Shock 37: 392-398, 2012.

4. Ueda T, Takeyama Y, Yasuda T, et al: Vascular endothelial growth factor increases in serum and protects against the organ injuries in severe acute pancreatitis. J Surg Res 134: 223-230, 2006.

5. Mura M, dos Santos CC, Stewart D and Liu M: Vaseular endothelial growth factor and related moleeules in acute lung injury. J Appl Physiol (1985) 97: 1605-1167, 2004.

6. Abadie Y, Bregeon F, Papazian L, et al: Decreased VEGF concentration in lung tissue and vascular injury during ARDS. Eur Respir J 25: 139-146, 2005.

7. Földi M and Zoltán OT: Unconditioned reflex activity in experimental lymphostatic encephalopathy and the therapeutic action of coumarin from Melilotus officinalis. Arzneimittel-Forschung 20: $1623-1624,1970$.

8. Liu MW, Su MX, Wang YH, Wei W, Qin LF, Liu X, Tian ML and Qian CY: Effect of melilotus extract on lung injury by upregulating the expression of cannabinoid $\mathrm{CB} 2$ receptors in septic rats. BMC Complement Altern Med 14: 94, 2014.

9. Sonkodi S: Decrease of spontaneous motility in experimental lymphogenic encephallopathy and the protective activity of coumarin from Melilotus officinalis. Arzneimittel-Forschung 20 (Suppl 11a): 1617, 1970 (In German).

10. Macias FA, Simonet AM, Galindo JCG, Pacheco PC and Sanchez JA: Bioactive polar triterpenoids from Melilotus messanensis. Phytochemistry 49: 709-717, 1998.

11. Szapiel SV, Elson NA, Fulmer JD, Hunninghake GW and Crystal RG: Bleomycin-induced interstitial pulmonary disease in the nude, athymic mouse. Am Rev Respir Dis 120: 893-899, 1979.

12. Mikawa K, Nishina K, Takao Y and Obara HA: ONO-1714, a nitric oxide synthase inhibitor, attenuates endotoxin-induced acute lung injury in rabbits. Anesth Analg 97: 1751-1755, 2003.

13. Seely KA, Holthoff JH, Burns ST, et al: Hemodynamic changes in the kidney in a pediatric rat model of sepsis-induced acute kidney injury. Am J Physiol Renal Physiol 301: F209-F217, 2011.

14. Mancuso P, Whelan J, DeMichele SJ, Snider CC, Guszcza JA, Claycombe KJ, Smith GT, Gregory TJ and Karlstad MD: Effects of eicosapentaenoic and gamma-linolenic acid on lung permeability and alveolar macrophage eicosanoid synthesis in endotoxic rats.Crit Care Med 25: 523-532, 1997.
15. Liu MW, Wang YH, Qian CY and Li H: Xuebijing exerts protective effects on lung permeability leakage and lung injury by upregulating Toll-interacting protein expression in rats with sepsis. Int J Mol Med 34:1492-504, 2014

16. Castanares-Zapatero D, Bouleti C, et al: Connection between cardiac vascular permeability, myocardial edema, and inflammation duringsepsis: role of the $\alpha 1 \mathrm{AMP}$-activated protein kinase isoform. Crit Care Med 41: e411-e422, 2013.

17. Fisher BJ, Kraskauskas D, Martin EJ, et al: Mechanisms of attenuation of abdominal sepsis induced acute lung injury by ascorbic acid. Am J Physiol Lung Cell Mol Physiol 303: L20-L32, 2012.

18. Skóra J, Barć P, Pupka A, et al: Transplantation of autologous bone marrow mononuclear cells with VEGF gene improves diabetic critical limb ischaemia. Endokrynol Pol 64: 129-138, 2013.

19. van der Flier M, van Leeuwen HJ, van Kessel KP, et al: Plasma vascular endothelial growth factor in severe sepsis. Shock 23: 35-38, 2005.

20. Yano K, Liaw PC, Mullington JM, et al: Vascular endothelial growth factor is an important determinalt of sepsis morbidity and mortality. J Exp Med 203: 1447-1458, 2006.

21. Wei J, Huang X, Zhang Z, et al: MyD88 as a target of microRNA-203 in regulation of lipopolysaccharide or Bacille Calmette-Guerin induced inflammatory response of macrophage RAW264.7 cells. Mol Immunol 55: 303-309, 2013.

22. Huang Y, Nikolic D, Pendland S, Locklear TD and Mahady GB: Effects of cranberry extracts and ursolic acid derivatives on P-fimbriated Escherichia coli, COX-2 activity, pro-inflammatory cytokine release and the $\mathrm{NF}-\kappa \mathrm{B}$ transcriptional response in vitro. Pharm Biol 47: 18-25, 2009.

23. Ghose R, Guo T, Vallejo JG and Gandhi A: Differential role of Toll-interleukin 1 receptor domain-containing adaptor protein in Toll-like receptor 2-mediated regulation of gene expression of hepatic cytokines and drug-metabolizing enzymes. Drug Metab Dispos 39: 874-881, 2011

24. Oltean S, Neal CR, Mavrou A, et al: VEGF165b overexpression restores normal glomerular water permeability in VEGF164-overexpressing adult mice. J Am Soc Nephrol 21: F1026-36, 2012.

25. Lucas R, Sridhar S, Rick FG, et al: Agonist of growth hormone-releasing hormone reduces pneumolysin-induced pulmonary permeability edema. Proc Natl Acad Sci USA 109: 2084-2089, 2012.

26. Russell JA: Management of sepsis. Minerva Med 99: 431-458, 2008.

27. Schick M, Isbary T, Schlegel N, et al: The impact of crystalloid and colloid infusion on the kidney in rodent sepsis. Intensive Care Med 36: 541-548, 2010.

28. Schlegel N, Baumer Y, Drenckhahn D and Waschke J: Lipopolysaccharide-induced endothelial barrier breakdownis cAMP dependent in vivo and in vitro. Crit Care Med 37: 1735-1743, 2009.

29. Kaphle K, Wu LS, Yang NY and Lin JH: Herbal medicine research in Taiwan. Evid Based Complement Alternat Med 3: 149-155, 2006.

30. Trouillas P, Calliste CA, Allais DP, et al: Antioxidant, anti-inflammatory and antiproliferative properties of sixteen water plant extracts used in the Limousin countryside as herbal teas. Food Chemistry 80: 399-407, 2003.

31. Zhao L, Tao JY, Zhang SL, et al: Inner anti-inflammatory mechanisms of petroleum ether extract from Melilotus suaveolens Ledeb. Inflammation 30: 213-223, 2007.

32. Gebre-Mariam T, Asres K, Getie M, et al: In vitro availability of kaempferolglycosidesfrom cream formulationsof methanolic extractof the leaves of Melilotus elegans. Eur J Pharm Biopharm 60: 31-38, 2005

33. Asres K, Gibbons S, Hana E and Bucar F: Anti-inflammatory activity of extracts and a saponin isolated from Melilotus elegans. Pharmazie 60: 310-312, 2005.

34. Guo X, Pan Y, Xiao C, et al: Fractalkine stimulates cell growth and increases its expression via NF- $\kappa \mathrm{B}$ pathway in RA-FLS. Int J Rheum Dis 15: 322-329, 2012. 